\title{
Do adjustments in search behavior depend on the precision of spatial memory?
}

\author{
G. Pfuhl • L. B. G. Barrera • M. Living • R. Biegler
}

Published online: 11 August 2012

(C) Psychonomic Society, Inc. 2012

\begin{abstract}
Various forms of uncertainty are important for decision making. How aware are we of the precision of knowledge, and how accessible it is? In three experiments, an assessment of the precision of spatial memory was needed to make optimal decisions. First, we examined search strategies in a search task in which the most efficient strategy was to head to one side of the target by a margin depending on the precision of spatial information, the "where to start" task. We found that nine out of of our 20 human subjects adapted the margin according to precision. Second, we let the subjects search for the location of a sample picture. On one-third of the trials, the target was not present, making it a "when to stop searching" task. We found that the subjects did not adjust their investment in search according to their precision. In the third experiment, we looked at whether there was transfer between the two tasks. Subjects who had been reminded of the relevance of uncertainty by the "where to start" task increased their search effort more in the "when to stop searching" task. Thus, the results show that the use of information about precision is not automatic, but can be triggered.
\end{abstract}

Keywords Uncertainty · Navigation · Comparative cognition · Confidence $\cdot$ Decision making $\cdot$ Spatial learning · Metacognition $\cdot$ Working memory $\cdot$ Human learning

G. Pfuhl $(\bowtie) \cdot$ L. B. G. Barrera $•$ M. Living $\cdot$ R. Biegler Psykologisk Institutt, Norges Teknisk-Naturvitenskapelige Universitet (NTNU), 7491 Trondheim, Norway

e-mail: gerit.pfuhl@gmail.com

R. Biegler

e-mail: Robert.Biegler@svt.ntnu.no
Animals and humans rarely have absolutely reliable and precise information, partly because both available information and processing are noisy, and partly because it is often in the interest of other organisms not to provide reliable and precise information (Glimcher, 2003, 2005). If cognitive systems must operate in a world of uncertainty, the study of cognition must attend to the degrees of reliability and precision of cues. Here we ask what role precision has in cognition. More specifically, how easily can information regarding the precision of spatial memory be accessed and used?

The forgetting of discrete items from working memory can be modeled in an all-or-none fashion: When an incoming new item exceeds memory capacity, a random old item drops out (Brown, 1992; Glassman, Garvey, Elkins, Kasal, \& Couillard, 1994; Zhang \& Luck, 2008). When remembering continuously variable information, memory of the stimulus is better described as a probability distribution, and forgetting as an increase in the variance of that distribution (for a thorough theoretical treatment, see White \& Wixted, 1999; for empirical data, see Biegler, McGregor, Krebs, \& Healy, 2001; Cheng, Collett, Pickhard, \& Wehner, 1987; Perkins \& Weyant, 1958; Thomas \& Lopez, 1962). If a probability distribution represents a memory, the variance of that distribution is needed to assess how well something is remembered. Precision is the inverse of the variance.

Many decision processes benefit from taking into account the variance inherent in a representation (Pfuhl, Tjelmeland, \& Biegler, 2011; Pfuhl, Tjelmeland, Molden, \& Biegler, 2009). However, theoretical benefits do not guarantee widespread use of estimates of precision. Trommershäuser, Maloney, and Landy (2008) reviewed evidence that perceptual and motor systems differ markedly from those involved in economic decision making. Perceptual and motor systems generally at least approximate statistically optimal decision making, yet humans clearly deviate from statistical 
optimality when making economic decisions that have the same mathematical description.

We suggest two possible reasons for this difference. One possible reason is that perceptual information and its variance are naturally represented as a distribution of neural activity (Knill \& Pouget, 2004; Pouget, Dayan, \& Zemel, 2003; Sahani \& Dayan, 2003), while the variance relevant to economic decisions or conditioning has to be constructed from memories of separate experiences. Furthermore, perceptual decision tasks are implicit, with only the final output made explicit, whereas economic tasks operate with explicit information at the input and output levels.

The second reason is that early perceptual processes typically deal with one stimulus dimension at a time. The variance then applies to that one dimension. In contrast, the content of a memory may be multimodal. Would there have to be a separate variance for each of a memory's elements, or can there be a common metric for the whole?

The question of whether there would be a common metric for the variance of different pieces of information is relevant whenever information from different sources is combined. In the cases we know in which cue combination takes into account variance, there clearly is a common metric. For example, Atkins, Fiser, and Jacobs (2001) studied the combination of haptic, textural, and motion cues into a depth estimate. Depth is the common metric, and whatever the variance in speed of motion or textural gradient might be, it needs to be translated into a variance in the corresponding depth estimate to allow statistically optimal cue combination. The same observation applies to the studies of Ernst (2007), Ernst and Banks (2002), Körding and Wolpert (2006), and Roach, Heron, and McGraw (2006). In these cases, it is known in advance what the potentially relevant information is and what the common metric is.

It is not clear to us whether that must apply to later stages of processing. If it is not possible to translate remembered variance into a common metric of a novel combination, the variance may not be represented in the first place; that is, only the value but not its precision gets remembered. Then, variance would not be available even for decisions that do use the same metric. If the same remembered information is used for two different decisions, both requiring access to variance for an optimal decision, will both decision processes have the same degree of access? Could one task prime access for the other?

We present here two tasks that allowed us to identify decision strategies that rely on knowledge of the precision of spatial memory: an edge-following task, in which the optimal starting point for search depends on how precisely the target location is known, and an analogue of a cache retrieval task, in which the optimal level of investment in search also depends on how precisely the target location is known. The cache retrieval task is a special case of the more general problem of how much effort to invest in an activity when feedback regarding the outcome is delayed. We first present the general logic of the two tasks, and then describe the experiments.

\section{Where to start a search: the error compensation strategy in an edge-following task}

In some situations, a position estimate may be more precise in one dimension than in others. For example, when a vessel approaches a harbor on a straight coast, there is no uncertainty about how far the harbor is from the coast, and once the vessel reaches the coast, there is no uncertainty about how far it is from there. But there may be uncertainty about how far the vessel is from the harbor. If, on sighting land, the harbor is not visible due to a navigational error, should the vessel turn right or left? A solution used for centuries, and still practiced by those without electronic navigation aids, is to head for one side of the coast by a margin exceeding the navigational error expected from previous experience navigating under similar conditions (see also Gatty, 1999, p. 78).

This error compensation strategy is more efficient than the alternative, the target-centered strategy of starting search at the most likely target location (Pfuhl et al., 2011). Assume that a normal distribution describes your knowledge of the target location. First consider the target-centered search strategy. Start search at the most likely location, the center of the distribution (Fig. 1a). If you do not find the target right there, you can turn right or left to continue your search. Having started in the center of a symmetrical distribution, you have no reason to prefer one direction over the other, so you pick one at random. As you search in one direction, the probability of finding the target in that direction decreases steadily. At some point, you are better off turning back and searching in the other direction, despite the cost of backtracking over the area you have already searched. With the parameters used for Fig. 1, that happens after having searched only $24 \%$ of the original probability distribution (Fig. 1c and e). If you do not find the target in the new direction, eventually reversing direction becomes once more worthwhile. You do that after having searched an additional $43 \%$ of the original probability distribution, for a total of $67 \%$.

By comparison, if you use the error compensation strategy with the same search parameters, the optimal point for beginning a search is 0.75 standard deviations off to one side of the center of the distribution (Fig. 1b). Turning back becomes worthwhile only after having searched $71 \%$ of the original distribution (Fig. 1f). In over two-thirds of searches you will find the target without ever turning back, and hence you will travel less. 

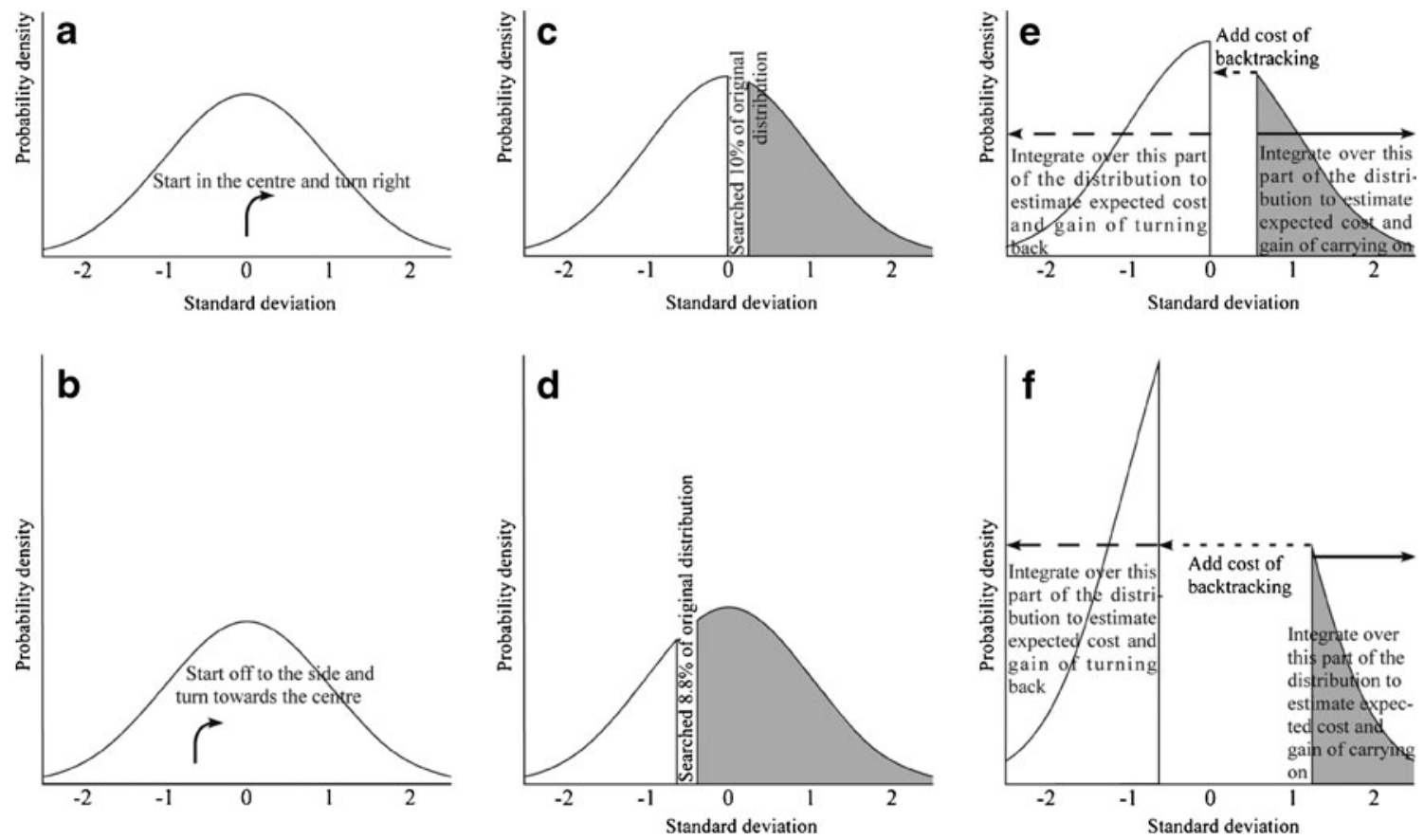

Fig. 1 Efficiency of the target-centered $(\mathbf{a}-\mathbf{c}-\mathbf{e})$ and error compensation (b-d-f) search strategies. If the location of an object is continuously variable, knowledge of the location is best described as a probability distribution. (a) The target-centered search strategy is to begin search at the most likely location of the target, in the center of the distribution. If the target is not found immediately, search continues in a randomly chosen direction, because there is no reason to prefer either side. (b) In contrast, the error compensation strategy involves beginning search off to the side from the center of the distribution by a margin that depends on the width of the distribution, the costs of search and travel, and the value of the target. If the target is not found immediately, it pays to turn toward the center of the distribution. (c and d) We assume that when the searcher reaches the target location, the target will not be overlooked. That means that if the target has not yet been found, it cannot be at locations that have already been searched. Therefore, the probability density distribution must be set to 0 for all of the locations that have been searched already. This has been done here for a distance corresponding to 0.25 standard deviations of the original distribution. If the target is known to be present, the rest of the distribution must be rescaled so that the area still adds up to 1. (e) At any moment when the target has not yet been found, the searcher must estimate whether it is more profitable to carry on searching in the same direction, or whether it is better to turn around, backtrack over locations that have already been searched, and carry on searching in the other direction. The expected gain and cost of carrying on searching can be estimated by integrating over the part of the distribution remaining in the same direction. The expected gain and cost of turning back can be estimated by the same integration over the remaining part of the distribution in the other direction and adding in the cost of backtracking. Optimal use of the target-centered strategy does depend on knowing the width of the distribution, even though the width has no influence on where search begins. (f) The same reasoning applies to the error compensation strategy. However, search proceeds for longer before the optimal point for turning back is reached. On average, the error compensation strategy results in longer search before turning back, less backtracking, and greater efficiency than the targetcentered strategy

\section{When to stop a search: cache retrieval}

Imagine a squirrel retrieving a cache of nuts in winter. (This was also the rationale given our subjects. Alternative scenarios are a favored food item in a full fridge or a paper on a desk.) At any point in time at which the squirrel has not yet found anything, it has to decide whether the failure so far means it has just not dug deep or wide enough, or whether it means that the cache has been pilfered and it would be better to search elsewhere. The computational task is similar to where to start a search: Estimate an initial probability distribution that represents your spatial memory of this location. During search, continuously update your estimate of the probability of the cache being there and how far you have to expect to search in order to find the cache if it is still there. As in "where to start a search," this involves integrating over probability distributions to estimate the expected costs.

A quantitative analysis of the relationship between estimated precision and optimal search radius is presented in Pfuhl et al. (2009). The following boundary conditions apply: If knowledge of the cache location is extremely precise - for example, the cache is almost certain to be within the area of a single probe - then such a single probe without finding the cache constitutes good evidence that the cache has been pilfered, even if the a priori probability of pilfering is very low. Therefore, one should stop searching after a single probe. If knowledge of the cache location is 
extremely vague, the expected cost of searching will exceed the utility of the cache, so one should never start searching. In between, the optimal limit to search effort will reach a maximum.

We first present an experiment establishing that external uncertainty influences search behavior in humans: the edgefollowing task. Next, a cache retrieval analogy in two dimensions is presented. Here we found that internal uncertainty did not influence search behavior. Finally, in the third experiment, we used a within-subjects design with a manipulation of internal uncertainty to see to what degree knowledge of precision is accessible in different situations.

\section{Experiment 1: edge following with external uncertainty}

The two search strategies described above should differ in several behavioral characteristics:

Direction of search If the target is not found immediately, subjects using the error compensation strategy should turn toward the target more often than chance. Subjects using the target-centered strategy should turn toward or away from the target equally often. This prediction applies for symmetrical search.

Distance between beginning of search and target If we can assume that the precision of spatial information is unrelated to the choice of strategy, then the distance between the point where search begins and the target should be greater when using the error compensation strategy than when using the target-centered strategy.

Reversing the direction of search Subjects using the error compensation strategy should turn back and search in the other direction less often than people using the targetcentered strategy. The distance between the beginning of search and the first reversal of direction, if any, should be greater when using the error compensation strategy.

Efficiency The error compensation strategy should be more efficient because it involves less backtracking over areas that have already been searched. Therefore, the total travel distance (search and backtracking) until finding the target should be less when using the error compensation strategy (Pfuhl et al., 2011). The reduced cost of backtracking means that the error compensation strategy should search a greater proportion of the original probability distribution before reaching the point where it is best to abandon search. The error compensation group should thus have a higher rate of success in finding the target.

Of these four behavioral characteristics, only two can be used to identify the search strategy: the direction of search and the dependence on the precision of the distance between the beginning of search and the target. Under realistic conditions, not enough information would be available to predict with sufficient accuracy the number of reversals of direction, the distance to the first reversal, or the efficiency of search. These measures could only be used to validate a classification based on the other criteria.

In Experiment 1, subjects carried out a spatial memory and search task on a computer screen. In addition to whatever variability in the estimation of the position of a target was due to forgetting, there was variability added by the program. In other words, we imposed external uncertainty - that is, uncertainty caused by variability in the environment-rather than internal uncertainty caused by ignorance (Kahneman, Slovic, \& Tversky, 1982).

\section{Method}

Subjects

The subjects were 20 students and staff recruited in spring and summer 1998 from the Department of Psychology, University of Newcastle, as well as the ICAPB and the Centre for Neuroscience, University of Edinburgh. Eleven of the subjects were female, 19-48 years of age, and nine were male, 19-55 years of age.

\section{Apparatus}

The apparatus was an Acorn A3000 microcomputer with a 14-in. color monitor, controlled by a program written in BBC BASIC.

\section{Procedures}

The subjects were shown a ring of 90 blue spots on a computer screen $27.5 \mathrm{~cm}$ wide and $20.2 \mathrm{~cm}$ high. One of those spots, chosen at random, flashed yellow for half a second. That was the sample stimulus that gave some information regarding the location of the target. A retention interval was filled by a distractor task-namely, clicking on a square that appeared seven times at random locations near the center of the screen. Next, the ring of 90 blue spots appeared again, and the task was to find the target location. After the first click on a spot, the display zoomed in by a factor of 3 , and the chosen spot was displayed under crosshairs. From there on, subjects had the choice to search the current location by clicking on the spot under the crosshairs, using the rightmost of three mouse buttons. They could choose to travel to a neighboring spot by clicking on that using the left mouse button, or to abandon search by holding down the middle mouse button and then pressing right and left 
buttons simultaneously. A spot that was searched and was not the target turned red. If a searched spot was the target, a short tone was played through the speakers, and the spot flashed red and white. Subjects were asked to minimize travel and search. To prevent subjects from reducing costs by searching only when they were very certain of the location of the target, we imposed a cost of giving up of 100 points, equivalent to traveling to and searching 50 spots. A random search would, on average, have to cover 45 spots, so giving up was only cost effective to prevent long backtracking.

Subjects searched for the target in each of four conditions, aiming for minimum cost when added up over all trials. There were ten trials in each of the four conditions, in random order. The location of the target was chosen randomly in each trial. In all conditions, the sample (yellow spot) gave information regarding the location of the target, but the precision of that information varied as follows:

1. The maximum distance between the sample and the target was zero. The target was exactly where the sample was.

2. The maximum distance between the sample and the target was one. The target might be exactly where subjects saw the sample, but it could also be one to the right or left.

3. The maximum distance between the sample and the target was three. The target might be exactly where subjects saw the sample, but it could also be up to three to the right or left.

4. The maximum distance between the sample and the target was six. The target might be exactly where subjects saw the sample, but it could also be up to six to the right or left.

This maximum difference between sample and target was written in the top left-hand corner of the screen, so subjects could always know how precisely the sample indicated the target location.

After the experiment, subjects were asked "What determined where your first choice was?" If they did not give an answer relevant to the purpose of the experiment, they were asked whether or not the first choice had been their best guess of the target location. On the basis of their answers, they were assigned to either the error compensation group or the target-centered group. Several subjects reported use of the compensation strategy only when the variability (maximum distance between sample and target) was at least 3 or at least 6 , only after initial use of the target-centered strategy, or both. These were all classified as using the compensation strategy. Any group differences are therefore conservative estimates, because the data from the compensation strategy group will include some trials in which subjects still used the target-centered strategy. One subject reported using the compensation strategy when the variability was at least three, except for two trials in which the sample was near the top or the bottom of the ring. These two trials were identified and excluded from the analysis.

Data analysis

The program recorded the following measures: direction of the second choice (with reference to the sample, not the target), the frequency of reversals, number of choices to reversal, and the distance of the first choice from the sample. Measuring the direction of the second choice was only possible if subjects did not find the target in the first choice. Reversals were only possible in trials with at least three choices. When variability was low, some subjects were accurate enough to provide only a few such trials. Therefore, these data were only analyzed across the whole experiment. Only distance between the first and second choices and total travel distance were examined for changes across trials. The data were analyzed using SPSS. When sphericity was significantly violated, we report the values from the Greenhouse-Geisser correction.

\section{Results}

Nine subjects (four were females) reported use of the compensation strategy for at least some trials. Three of them reported that they had initially used the target-centered strategy and only later switched to the compensation strategy. Furthermore, three of the nine subjects used the compensation strategy only when the variability was 6 , and a further two subjects also used the compensation strategy when the variability was 3 . Eleven subjects reported use of the target-centered strategy throughout the experiment.

A mixed analysis of variance (ANOVA) of the direction of the second choice of each trial showed that subjects in the error compensation group were more likely to move toward the sample after their first choice $[F(1,18)=11.68, p=.005$, $\eta^{2}=.393$; Fig. 2a]. Averaged across groups, subjects were not significantly more likely to move toward the target when variability was large [main effect of variability: $F(3,54)=$ $\left.2.75, p=.051, \eta^{2}=.133\right]$. However the error compensation group was more likely to move toward the target when variability was large [interaction between group and variability: $\left.F(3,54)=4.56, p=.006, \eta^{2}=.202\right]$.

The error compensation group showed a lower frequency of reversals than did the target-centered group $[F(1,18)=$ 20.71, $p<.0001, \eta^{2}=.535$; Fig. 2b]. The frequency of reversals decreased with increasing variability $[F(1.89$, $33.96=7.72, p<.01, \eta^{2}=.3$ ] , and there was no interaction.

The error compensation group started search farther away from the sample than did the target-centered group $[F(1,18)=$ 17.09, $p<.001, \eta^{2}=.487$; Fig. $\left.2 c\right]$. That distance increased 
Fig. 2 Differences between the error compensation and targetcentered search strategies. (a) Direction: The second choices of the error compensation group were directed toward the target more often than chance, and more often than in the targetcentered group when variability was greater than 0 . (b) Frequency of reversals: The error compensation group reversed the direction of search less than did the target-centered group, and for variabilities 3 and 6 , less than would be expected by chance. (c) Distance from first choice to target: For variabilities 3 and 6 , the error compensation group started search farther from the target. (d) Number of choices to reversal: Subjects in the error compensation group kept searching for longer before reversing direction than did subjects in the target-centered group. (e) The total travel distances in trials in which subjects found the target were equivalent across groups
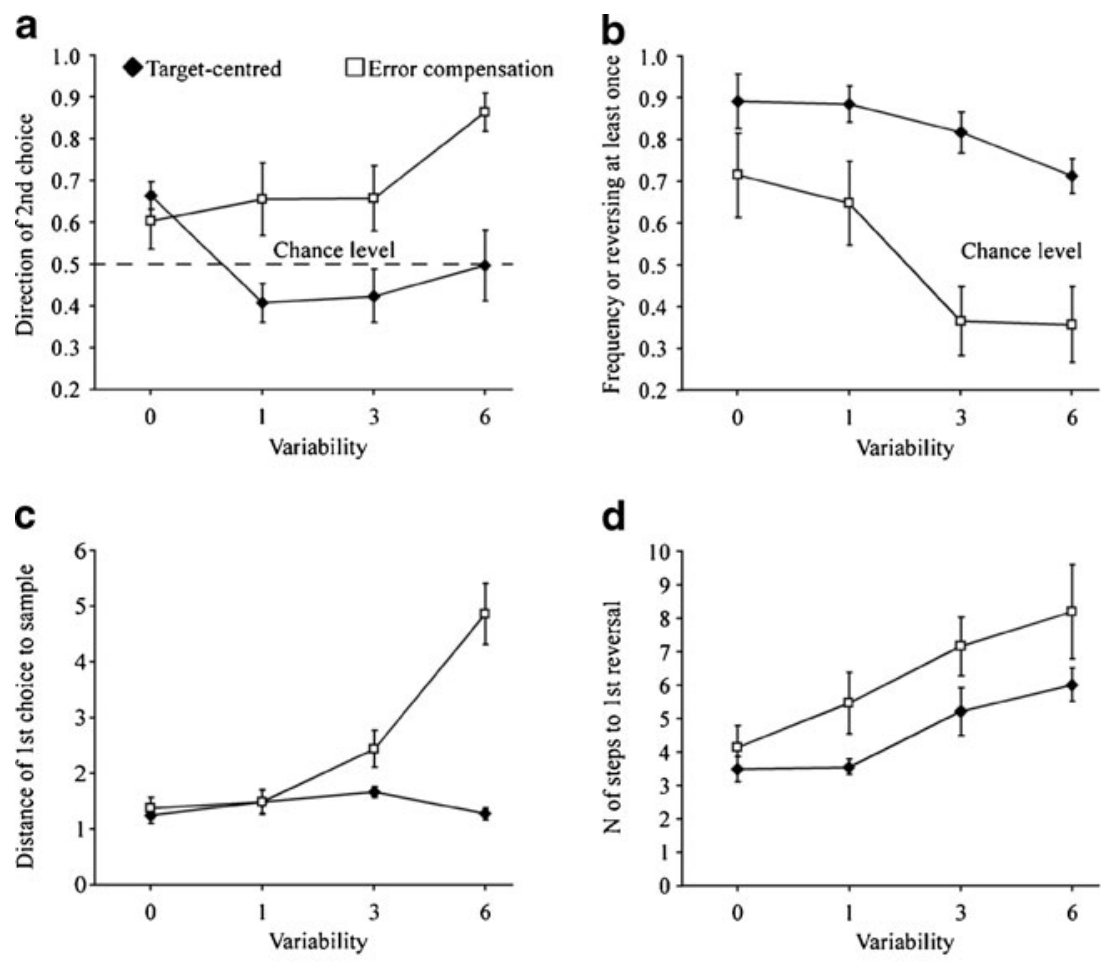

with variability $\left[F(3,54)=39.22, p<.0001, \eta^{2}=.685\right]$, and variability also interacted with group $[F(3,54)=43.94, p<$ $\left..0001, \eta^{2}=.709\right]$. The distance of the first choice from the sample changed as a function of variability in the error compensation group $\left[F\left(1.33,10.61=45.63, p<.0001, \eta^{2}=.851\right]\right.$, but not in the target-centered group $[F(3,30)=2.47, p=.081$, $\left.\eta^{2}=.198\right]$.

The error compensation group included subjects who only switched to that strategy partway through the experiment, and some subjects reported using the error compensation strategy only when variability was large. Were changes related to strategy choice greater in this group and when variability was high? The distance between first choice and sample increased from the first to the second half of the trials $\left[F(1,18)=20.86, p<.001, \eta^{2}=.537\right]$. An interaction of first versus second half with group $[F(1,18)=$ 10.54, $p<.001, \eta^{2}=.369$ ] was due to only the error compensation group increasing distance in the second half of trials $\left[F(1,24)=17.69, p<.01, \eta^{2}=.689\right]$, while the target-centered group did not change $[F(1,30)=1.78, p=$ $\left..212, \eta^{2}=.151\right]$. We found an interaction of first versus second half with variability $\left[F(3,54)=8.15, p<.001, \eta^{2}=\right.$ $.312]$, as well as a triple interaction of group, first versus second half, and variability $\left[F(1,30)=6.88, p<.001, \eta^{2}=\right.$ .277]. Separate analyses of each level of variability showed that interactions of group with first versus second half occurred only when the variability was $3[F(1,18)=8.99$, $\left.p<.01, \eta^{2}=.333\right]$ or $6\left[F(1,18)=16.95, p<.001, \eta^{2}=\right.$ $.485]$, but not when the variability was 0 or $1(F<1)$.

The number of steps to the first reversal was larger in the error compensation group $\left[F(1,17)=15.53, p<.002, \eta^{2}=\right.$ $.477]$ and grew with increasing variability $[F(3,51)=7.39$, $p<.001, \eta^{2}=.303$; Fig. 2d]. Group and variability did not interact $(F<1)$.

Finally, total travel distance increased with variability $\left[F(1.27,18.99)=33.32, p<.0001, \eta^{2}=.69\right]$. All other main effects and interactions failed to reach significance (Fig. 2e). 
Table 1 Predictions for and results of Experiment 1

\begin{tabular}{|c|c|c|c|c|}
\hline & \multicolumn{2}{|l|}{ Compensation strategy } & \multicolumn{2}{|l|}{ Target-centred strategy } \\
\hline & Prediction & Results & Prediction & Results \\
\hline \multirow{2}{*}{$\begin{array}{l}\text { Direction of 2nd } \\
\text { choice }\end{array}$} & Go towards sample & + & Random & $+($ except for variability $=0)$ \\
\hline & $\begin{array}{l}\text { More often towards sample } \\
\text { with increasing variability }\end{array}$ & + & $\begin{array}{l}\text { Independent of } \\
\text { variability }\end{array}$ & $\begin{array}{l}\text { Towards target when variability }=0 \text {, when there } \\
\text { often is a choice between only two alternatives }\end{array}$ \\
\hline $\begin{array}{l}\text { Frequency of } \\
\text { reversals }\end{array}$ & $0+$ unnecessary reversals & + & $\begin{array}{l}0.5+\text { unnecessary } \\
\text { reversals }\end{array}$ & + \\
\hline \multirow{2}{*}{$\begin{array}{l}\text { Distance 1st } \\
\text { choice to } \\
\text { sample }\end{array}$} & $>$ variability & + & $\begin{array}{l}\text { Small, determined only } \\
\text { by subjects' accuracy }\end{array}$ & + \\
\hline & Increases with variability & + & $\begin{array}{l}\text { Independent of } \\
\text { variability }\end{array}$ & + \\
\hline \multirow{2}{*}{$\begin{array}{l}\text { Number of steps } \\
\text { to } 1 \text { st reversal }\end{array}$} & $\geq 2 *$ variability & + & $\geq$ variability & + \\
\hline & $>$ target-centred group & + & $<$ compensation group & + \\
\hline
\end{tabular}

The large "+" signs stand for predictions that were fulfilled

\section{Discussion}

The results were generally consistent with our predictions as to how strategy choice affects behavior (see Table 1). Subjects using the compensation strategy were more likely to move toward the sample in their second choice than those using the target-centered strategy. The error compensation group also showed a lower frequency of reversals, a larger distance from the first choice to the sample, and a larger number of steps to reversal. Variability had an effect on the results in all four measures, and interacted with strategy choice as predicted. The exception was the efficiency of search. Since we imposed a 100-point punishment when abandoning search, we found that even subjects in the error compensation group backtracked over long distances.

We found that our characterization of the error compensation strategy was both internally consistent and accorded with the experimental results. Furthermore, it does not require navigational expertise to use information on the precision of spatial knowledge to determine parameters of a search strategy, as nine of our 20 subjects did so spontaneously. That makes it more plausible that the strategy may be used by other species as well—for example, ants (Wolf \& Wehner, 2005). In Experiment 1, subjects could use estimates of the precision of spatial information to decide where to start searching. In Experiment 2, we developed a task in which estimates of the precision of spatial memory could be used to decide when to stop searching. The procedures differed in several respects. In Experiment 3, we used the same procedures to present items for our subjects to remember and varied only whether the subjects were asked where to start or when to stop searching.

\section{Experiment 2: cache retrieval and recognition}

Subjects carried out a spatial memory and search task on a computer screen. The location of a briefly shown picture was to be remembered for later search. We performed two manipulations. First, in half of the trials, subjects were distracted by an addition task before they could start searching. The source of imprecision in spatial information was now internal, rather than external. Second, the picture could not be found in some trials (pilfering). In these trials, we could measure the maximum effort people were willing to invest in searching - that is, size of the search radius. We expected a larger search radius in trials with distraction, since the memory of the target location was less precise. Between groups, we manipulated the cost of searching - that is, how fast the subject made progress in searching an area.

We measured the precision of spatial memory by two different methods. On the one hand, we measured the distance between the target location and where the subjects started to search. On the other hand, we used forced choice recognition (signal detection theory) to see how accurately the location of the picture was remembered. The better the memory, the higher the $d^{\prime}$ - that is, discrimination between a picture that was shown at its original place versus a displaced picture. We used both methods in a within-subjects design.

\section{Method}

A group of 30 subjects (17 female: mean age $26.2 \pm$ 6.2 years, median 25 , range $20-47 ; 13$ male: mean age $25.8 \pm 5.2$ years, median 25 , range $19-37$ ) were recruited. All had normal or corrected-to-normal vision.

Materials and procedures

A computer program written with LabView 6.1 (National Instruments) displayed sample and choice stimuli on computer monitors measuring $32.8 \times 24.8 \mathrm{~cm}$. Before starting the experiment, subjects received an explanation and 
demonstration of the task. We told subjects that we were interested in spatial memory and in the efficiency of search. Subjects were told that in 16 of the 46 recall trials, they would not be able to find the target, no matter how long they searched. The subjects had ten practice trials before proceeding to the experimental trials. All trials began with a 1s-long presentation of a picture measuring $1 \times 1 \mathrm{~cm}$, chosen randomly from a set of 28 pictures, with the constraint that the picture from the immediately preceding trial was avoided. The pictures showed, for example, a hot air balloon, a sunflower, a space shuttle launch, a windsurfer, a tartan pattern, bluebells, mushrooms, and the like. The pictures were intended to be easy to discriminate, so as to reduce the chance that subjects would search at a location remembered from a previous trial, but otherwise picture identity was irrelevant. The picture was displayed anywhere on the screen, with the constraint that the center of the picture was at least three picture diameters from each edge and three picture diameters from the center of the screen. That gave 597,024 possible locations.

There were eight types of trials. The four types of recall trials differed in whether or not subjects had to complete a distractor task during the retention interval and in whether subjects could find the target picture (30 trials) or not (16 trials). The four types of recognition trials (10 trials of each type) also differed in whether or not subjects carried out the distractor task and in whether the picture reappeared at the same or a different place. These were all within-subjects factors. Search cost in recall trials (see below) was a between-subjects factor. The order of trials was random, with the constraint that there would be no more than three trials in a row of the same type. This ensured that a subject could not know in advance whether in the choice phase one had to recognize or recall the location and whether the target could or could not be found.

Retention interval and distractor task, for both recall and recognition trials On half of the trials, subjects were not asked to do anything during the 3-s retention interval. On the other half of the trials, they were presented with an addition task as a distractor. During the distractor task, a new window covered the screen. A series of random numbers ranging from 0 to 9 was presented at random locations on the screen. The subjects' task was to add up these numbers. Four numbers were presented at an initial rate of one per second. The presentation rate was titrated, with the interval decreasing by $30 \mathrm{~ms}$ after each correct judgment and increasing by $90 \mathrm{~ms}$ after each error. A fifth number was displayed if the presentation rate increased enough that the fifth number could be put on the screen before $3 \mathrm{~s}$ had elapsed since the beginning of the retention interval. Then a number was presented in the center of the screen, flanked by a green button marked "TRUE" and a red button marked
"FALSE." With equal probabilities, this number was either the sum of the preceding numbers, in which case subjects should click on the "TRUE" button, or it was up to two more or two less, in which case subjects should click on the "FALSE" button. These buttons were displayed until either the subject made a choice or $3 \mathrm{~s}$ had elapsed, after which the window closed. If the subject had not made a choice, an error was registered. Trials with and without the distractor task were randomly ordered.

Search phase, recall trials A copy of the stimulus picture was presented in the center of the screen, with a green "Next trial?" button underneath. This reminded subjects of which picture's location they were supposed to remember, and it was intended to reduce the probability of subjects searching at a location where they had seen the picture in a previous trial. Once subjects had been prompted by the picture in the center, they could start searching by clicking at the location where they thought they had seen the picture during the sample phase. Holding down the left mouse button expanded a circle. Once a search had started, the search location could not be corrected. Search could be interrupted at any point by releasing the mouse button. Search continued from the same location until a subject either found the target picture or abandoned search by clicking on the "Next trial?" button. On trials in which subjects could find the target (30 out of 46 trials), the circle disappeared as soon as one of its edges reached the coordinate where the center of the sample picture had been. This automatically terminated the trial.

The expansion speed of the search circle was such that the total area was (a) proportional to the square root of total search time (decelerating function), (b) proportional to total search time, or (c) proportional to the square of the total search time (accelerating function). All search functions reached the same area of $10.84 \mathrm{~cm}^{2}$ after $6 \mathrm{~s}$. The speed at which the search circle expanded was controlled by changing the time intervals between successive increases of the radius by one pixel. The linear function was implemented by making the interval proportional to the difference in areas between successive circles (in retrospect, an unnecessarily complicated way to make the interval proportional to radius). The accelerating function was implemented by keeping the interval constant. The decelerating function was implemented by making the interval proportional to the area of the circle.

Subjects were asked to maximize the rate at which they could find target locations. After each trial, during the 2-s intertrial interval, that rate was displayed, as a number with one decimal point, in the form of targets found per minute total search time.

The expanding search circle is a reasonable approximation for searches such as digging a hole to retrieve a cache, making many probes spaced closely enough that the target item will not be missed in a gap between probes, or for a 
search spiral with navigational error that is small as compared to the spacing of the spiral. Furthermore, we assumed that search is perfectly effective, meaning that search would never miss the target. Therefore, if the target had not been found within the search area, it was certain that the target was not within that area. These two assumptions are consistent with those in our mathematical analysis (Pfuhl et al., 2009). If the probability of detecting the target when coming across it is low, or navigation inaccurate, other search patterns may be preferable (Wehner \& Srinivasan, 1981).

Forced choice recognition task On half of the trials after the retention interval, the sample picture appeared again on the screen either at its original location or $2 \mathrm{~cm}$ (twice the picture size) away in a random direction. In the center of the screen were the buttons "same place" in blue and "different place" in red. These buttons were displayed until the subject made a choice.

Data analysis

On recall trials, we recorded the deviation between the target location and the subject's first choice (measurement of precision), as well as the search radius (Fig. 3). The data were recorded for each trial but were averaged per subject for further analysis, unless mentioned otherwise. To estimate the investment in search, we used only those trials in the cache retrieval task on which the subjects gave up, which were at least all trials without chance of finding the target (16 out of 40). In recognition trials, we classified responses as true and false positives and true and false negatives. We used the numbers of those responses to calculate $d^{\prime}$, the performance measure derived from signal detection theory (Macmillan \& Creelman, 1991), for each subject. We used the convention that a perfect score, which would make $d^{\prime}$ infinitely large, was avoided by adding 0.5 as the $(n+1)$ th choice.

\section{Results}

Forced choice recognition

Subjects discriminated better between familiar and new picture locations in the without-distractor trials than in the with-distractor trials: without distractor $d^{\prime}=2.52(S D=$ $0.79)$, with distractor $d^{\prime}=1.05(S D=0.68) ; F(1,27)=$ $31.14, p<.0001, \eta^{2}=.72$. This confirms that the distractor task reduced the precision of spatial memory. As expected, the cost of search had no effect on the precision of memory $(F<1)$, nor was there any interaction $(F<1)$.

Search in recall trials

The distractor task decreased the precision with which subjects remembered the target location. After the distractor task, the points where search began were more widely scattered, producing a greater standard deviation of the search coordinates $\left[F(1,27)=96.47, p<.001, \eta^{2}=.78\right]$. The cost of search did not affect the precision of memory $(F<1)$, nor did cost interact with distraction $(F<1$; Fig. 4$)$.

The $d^{\prime}$ of the forced choice recognition task and the standard deviation correlated significantly $[r(60)=.596, p<.0001]$. This correlation is important, because it validates our measure of precision by the standard deviation of the search coordinates.

The search radius did not change "with" or "without" the distractor task, regardless of the cost function used, nor was there an interaction between search radius and cost function

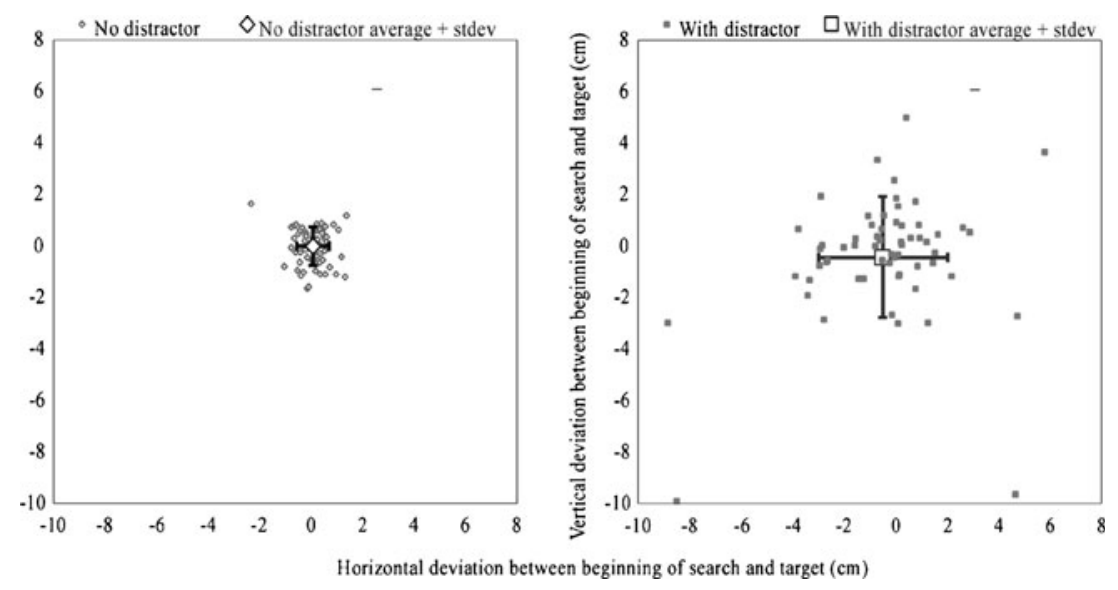

Fig. 3 Measurement of scatter. On each trial, we registered where search began relative to the target location. We calculated the horizontal and vertical standard deviations of these relative search coordinates. The average of the horizontal and vertical standard deviations was then the overall standard deviation that we used as a measure of how widely scattered search coordinates were. The data shown here come from a randomly chosen subject in Experiment 2. Note that each coordinate comes from a different trial. These were no multiple probes within a single trial 


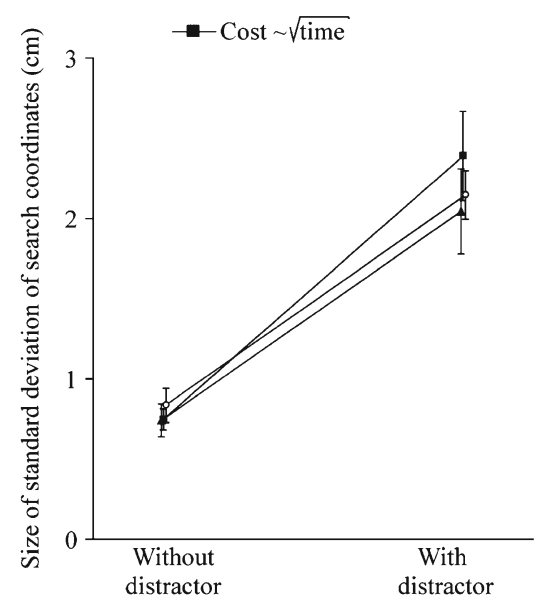

Fig. 4 Search scatter and search effort in Experiment 2. (Left) In trials with a distractor task, search coordinates were more widely scattered, as shown by greater standard deviations. The increase in scatter was independent of the cost of search. (Right) The search radius remained

(all $F_{\mathrm{S}}<1$ ). This failure to adapt the search radius according to the precision of memory was surprising.

We found that a low cost function did lead to longer searches than did more costly search functions $[F(2,27)=$ 22.04, $\left.p<.001, \eta^{2}=.62\right]$. Post-hoc Tukey tests confirmed that the lowest-cost function - that is, the one in which the radius was proportional to search time-was different from the other two cost functions (Fig. 4).

\section{Discussion}

The cache retrieval task employed here shows that the precision with which subjects remember the location of an item can be manipulated with a distractor task. However, the reduced precision did not result in prolonged search; the search radii were similar for trials with and without the distractor task. It is not clear whether subjects were not aware of remembering the target location less precisely after being distracted, or whether they used a rule of thumb and internal time limit for searching. Whether the cost of search per area decreased, remained the same, or increased affected only the total investment in search. However, search was independent of the precision of memory for all cost functions. Thus, we have no evidence that humans are well calibrated to optimize search in this setting. The question remains whether humans simply do not optimize search at all, or whether there was a problem with the experimental setting.

\section{Experiment 3: access to precision between tasks?}

There were three differences between Experiments 1 and 2: the nature of the search task, the source of imprecision of

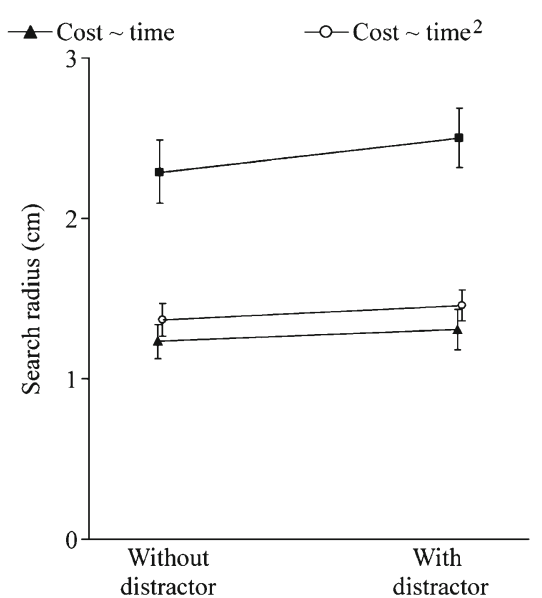

the same in the conditions with and without a distractor task. When search costs decreased (cost proportional to the square root of search time), subjects searched longer in general, but were still not affected by the distractor condition

spatial information, and the shape of the probability distribution that described spatial information. The logical next step was to test which of these factors was responsible for imprecision affecting search in Experiment 1 but not in Experiment 2. In error compensation, the underlying computational task is to assess imprecision (e.g., how wide is that probability distribution?), estimate the average effort to find the target when starting at some point of the probability distribution, and then optimize that function. In the case of internal uncertainty, the probability distribution is more likely to be normal, rather than the rectangular distribution of external variability in Experiment 1.

About half of the subjects in Experiment 1 had used information on external uncertainty to determine where to start searching, yet in the cache retrieval task of Experiment 2 , internal uncertainty had no influence on search. Could our subjects judge only external, but not internal uncertainty? Or did the cache retrieval task fail to trigger access to an estimate of internal uncertainty, at least in a small-scale computer-based task? By using a one-dimensional version of the cache retrieval task, we could make the stimulus presentation and the manipulation of internal uncertainty identical in an edge-following and a cache retrieval task. Each subject carried out both tasks, with the order counterbalanced across subjects. If a general process underlies behavior in both tasks, there should be transfer between them.

\section{Method}

Subjects

The subjects were 64 females and 35 males. The women were $18-54$ years of age (average 24 , median 22), the men 
were 18-44 years of age (average 23.7, median 23). All had normal or corrected-to-normal vision.

The data from a further 11 subjects were excluded for the following reasons: Data saving did not work properly and we could not reconstruct the data unambiguously $(n=7)$, or no search was performed in distractor trials, the subject used a finger to mark target locations, search stopped after a single click, or the edge task repeated four times $(n=1$ apiece). In total, 70 of the subjects took part in the experiment as part of their course requirements, and the rest were asked whether they would volunteer.

Materials and procedures

A computer program written with LabView 6.1 (National Instruments) displayed the sample and choice stimuli on computer monitors measuring $32.8 \times 24.8 \mathrm{~cm}$.

Instructions to subjects

Before starting the experiment, the subjects received an explanation and demonstration of their first task. We told subjects that we were interested in spatial memory and in the efficiency of search.

Subjects then had ten practice trials, before proceeding to 40 experimental trials. After a subject had finished these, we explained the other task, and subjects carried out another ten practice trials and 40 experimental trials. A total of 52 subjects started with the edge-following task, and 47 with the cache retrieval task.

To explain the concept of efficiency in the cache retrieval task, we offered the example of a squirrel that has buried nuts in autumn and attempts to recover them in winter. If it digs where it cached nuts and fails to find them, it must decide whether it is not quite in the correct spot and it is worth searching further, or whether a mouse might have found and eaten the nuts two months earlier, and it would be better to search elsewhere. We explicitly told subjects that on 16 of the 40 trials they would not be able to find the target, that this was analogous to the squirrel's cache having been pilfered, and that identifying these trials and not wasting effort on them was part of their task. We emphasized the importance of efficiency, of finding the maximum number of nuts per unit of search effort. In our description of the task, we carefully avoided the use of terms with negative connotations, such as "quitting," "giving up," or "abandoning" search.

When explaining the edge-following task, we said that it was analogous to forgetting sunglasses on a beach and going back to retrieve them. One of the features of such a search is that if one does not find the correct spot immediately and goes off in the wrong direction, it may be necessary to backtrack over places already searched.
Procedure

Sample phase All trials began with the presentation of a picture measuring $1 \times 1 \mathrm{~cm}$, chosen randomly from a set of 28 pictures (just as in Exp. 2), with the constraint that the picture from the immediately preceding trial was avoided. Because we needed both search tasks to be onedimensional, each sample picture was presented on a blue horizontal line. On the first trial, that line moved down one seventh of the vertical size of the screen from the top. On each of the next five trials the line moved down one seventh, then that sequence started again from the top. This was intended to reduce proactive interference among trials. The stimulus pictures were presented at random locations along the line but only within the central $70 \%$ of that blue line, so as to reduce edge effects (subjects were not told of this constraint; for bias toward borders, see Huttenlocher, Hedges, \& Duncan, 1991).

Retention interval and distractor task These aspects were the same as in Experiment 2.

Search phase An empty blue line appeared at the same height as during the sample phase, and a copy of the stimulus picture was presented in the center of the screen, with a green "Next trial?" button underneath. The difference between the cache retrieval and edge-following tasks lay in the ways that subjects could search for the target (see below).

Subjects would earn 5 points for finding the target, minus the search cost. That cost was proportional to search distance and was independent of time. The current search cost was displayed in the upper left corner of the screen. At the end of a trial, the current total sum of points from all trials was displayed in the center of the screen during the 2 -s intertrial interval.

Cache retrieval task In the cache retrieval task, a red rectangle expanded horizontally from the $x$-coordinate of the first click (Fig. 5a-c). On trials in which subjects could find the target (24 out of 40 trials), the red rectangle disappeared as soon as one of its edges reached the coordinate where the center of the sample picture had been.

Edge-following task In the edge-following task, the first click established the starting point of search. A click to one side would let a rectangle expand from the starting point toward the chosen side only (Fig. 5a and d-f). It was possible to reverse the direction of search by clicking on the other side of the rectangle. This created a new rectangle of a different color, expanding in the other direction and covering the rectangle already there. In other words, the new search first had to backtrack over an area that has already been searched. The search cost was proportional to the total travel distance, including travel over what has already been searched. 

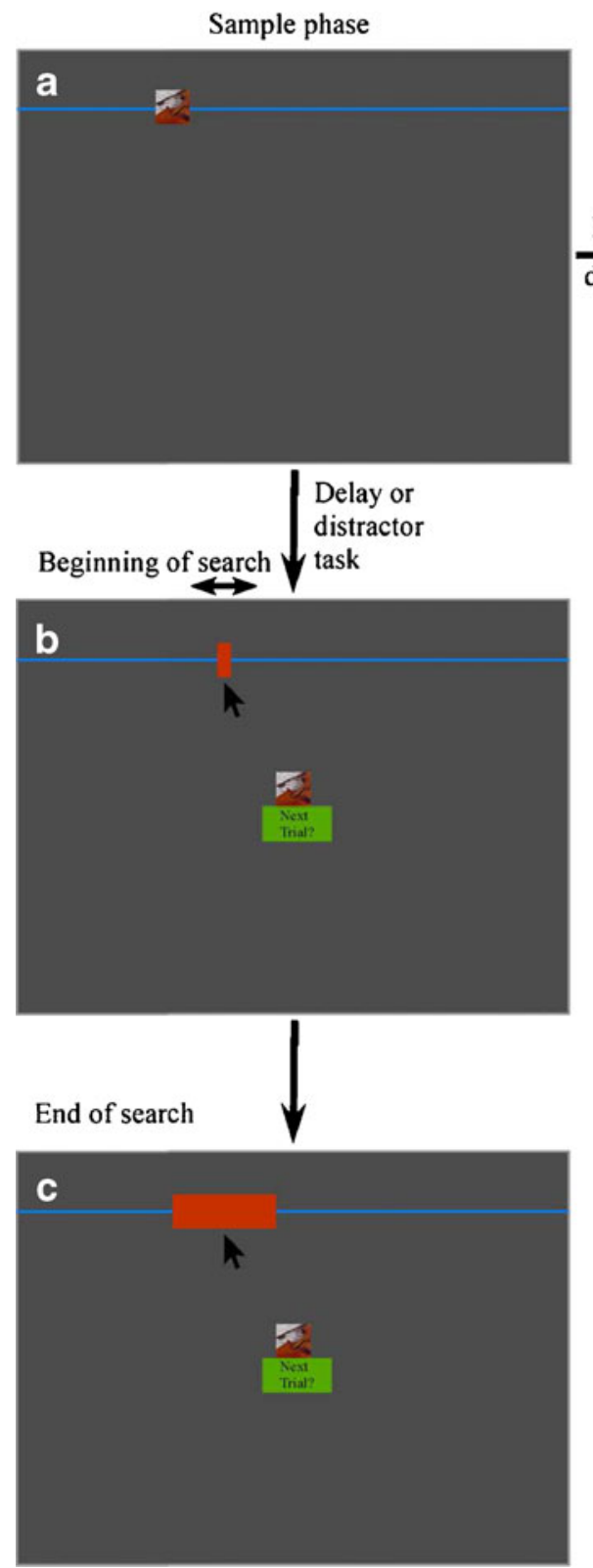

Fig. 5 Experiment 3: Sample and search phases in the cache retrieval task (a-c) and in the edge-following task (a and d-f). (a) A sample picture was presented at a random location within the central $70 \%$ of the blue line. In the next trial, the line would move to the next position down out of seven, then again to the upper position shown here. (b) The search area, marked by a red rectangle, expanded in both directions from the start point for as long as the subject held down the left mouse button. The search cost was proportional to the length of the rectangle and was shown in the upper left corner of the screen (omitted here because it would be too small to read if shown to scale). (c) On trials in which the target was present, search ended when the edge of the search area reached the point where the center of the sample picture

\section{Data collection}

After the experiment, subjects were asked how they had determined where to start search in the edge-following
Beginning of search
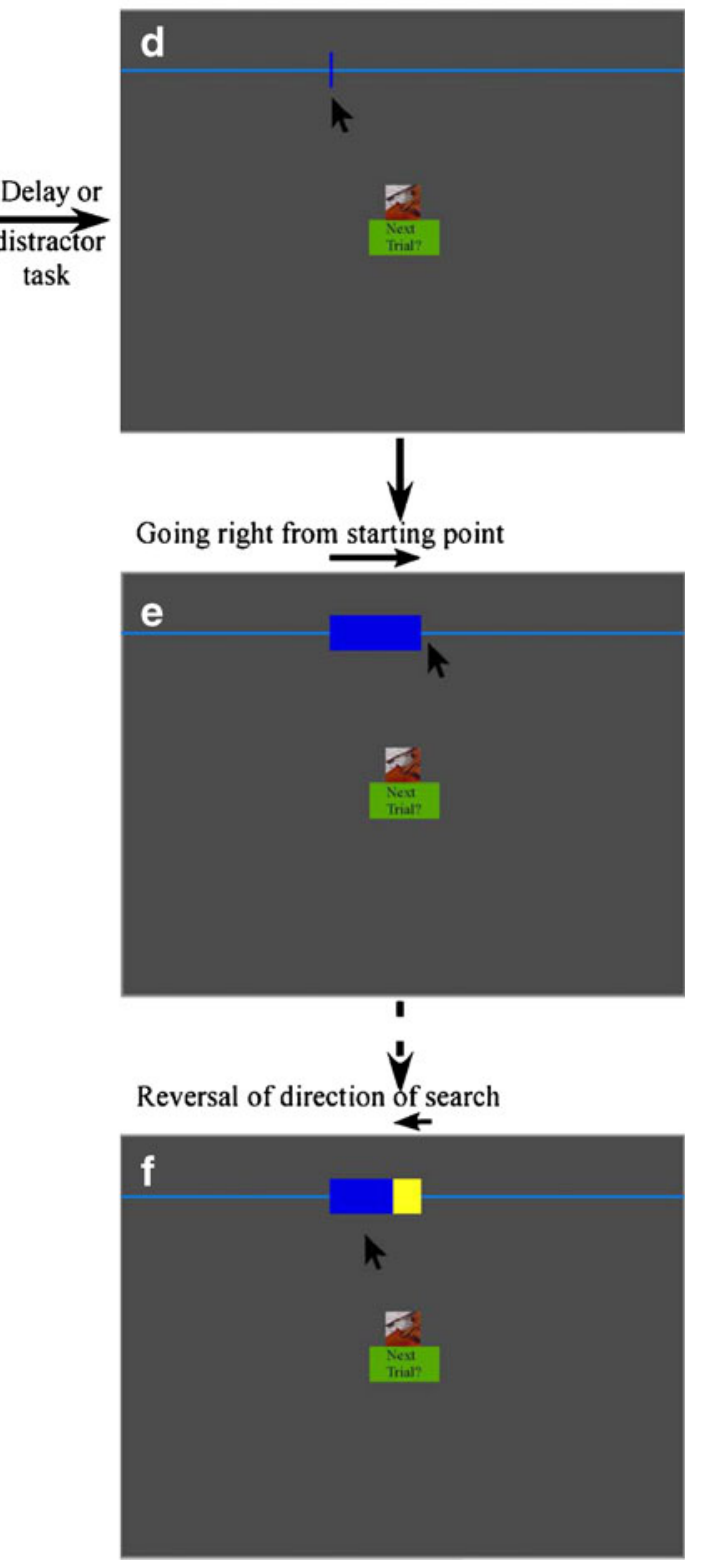

was. On trials in which the target was not present, search ended only when the subject clicked on the green button marked "Next trial?" (d) In the edge-following task, the search area expanded in only one direction. Shown here is the case in which the mouse pointer was moved to the right of the starting point. (e-f) The search area expanded toward the mouse pointer for as long as the subject kept the mouse somewhere beyond the expanding edge of the rectangle and held down the left mouse button. Moving the mouse to the other side of the expanding edge generated a new search area expanding from this new starting point. Movement to the left produced a rectangle marked in yellow. The search cost was proportional to the total travel distance, including backtracking over locations that had already been searched

task. If the reply referred to memorization strategies, the question was refined to how they had determined where to start searching once they had worked out where they thought the target was. 
For the edge-following task, we recorded the success rate, frequency of reversals, travel distance, distance between the target and the subject's first choice, whether the travel direction was toward the target, and distance to reversals. For analysis of the subjects' comparing strategies, we used the travel direction as an indicator of each subject's search strategy (see Exp. 1), and not the subjective reports. A subject was classified as target-centered if the travel direction scores in the with- and without-distractor conditions were below .75 or if the average among the two conditions was below 7 .

For the cache retrieval task, we recorded the deviation between the target location and the subject's first choice (measurement of precision), as well as the search radius. The data were recorded for each trial but were averaged per subject for further analysis, unless mentioned otherwise.

To estimate the investment in search, we used only those trials in the cache retrieval task on which the subjects gave up, which were at least all trials without a chance of finding the target (16 out of 40).

In both tasks, the occurrence of and performance on the distractor task were recorded, along with the times when the first and last choices were made. All proportions were arcsine-square-root transformed before analysis (Zar, 1999). The figures show untransformed data.

We were not interested in sex differences but in strategy (edge-following task) and order (cache retrieval task). However, a sex difference in strategy use was found. Sex was not entered as covariate in the analysis done below, as we were interested in the precision of memory, and that did not differ among the sexes (see the cache retrieval task below).

\section{Results}

\section{Choice of search strategy}

We first wanted to find out which subjects assessed the precision of spatial information in the edge-following task. In total, 24 of the 99 subjects reported use of the target-centered strategy, although only 20 met the criterion when we looked at the travel direction. The remaining subjects used the compensation strategy. The distribution of sex by strategies was uneven: Of the 20 target-centered subjects, only three were male. This sex difference in strategy choice was significant $\left[\chi^{2}(1,99)=4.94, p=.026\right]$.

Because precision was manipulated internally, we carried out an analysis of the four behavioral criteria listed above:

Direction of search The proportion of trials in which the target-centered group directed choices toward the target was at chance level, while the subjects in the error compensation group usually turned toward the target (Fig. 6a). The error compensation group more often turned toward the target $[F(1$, $\left.95)=156.56, p<10^{-21}, \eta^{2}=.62\right]$, which happened more often on trials without the distractor task $[F(1,95)=22.95, p=$ $\left..0001, \eta^{2}=.19\right]$. We also found an interaction between group and distractor task $\left[F(1,95)=4.45, p=.037, \eta^{2}=.04\right]$; that is, performance for the error compensation group decreased somewhat more with the distractor task.

Distance between beginning of search and target Because the target-centered group showed less precise memory (see the analysis of the cache retrieval task below), we could not predict which group would, on average, start search farther from the target; the greater random scatter of the targetcentered group could lead to the same or to a greater distance than the systematic deviation of the error compensation group. Because the necessary preconditions were not met, we did not analyze these data.

Reversing the direction of search The error compensation group reversed their direction of search less frequently than did the target-centered group $[F(1,95)=72.8, p<.0001$, $\left.\eta^{2}=.83\right]$. The distractor task increased the frequency of reversals $\left[F(1,95)=11.46, p<.001, \eta^{2}=.11\right]$, and there was no interaction $(F<1$, Fig. $6 b)$.

Efficiency First, we analyzed search distance and success rate separately. The total distance searched did not differ significantly between groups $\left[F(1,95)=3.4, p=.067, \eta^{2}=\right.$ $.03]$, although the distractor task did increase the distance $[F$ $\left.(1,95)=77.99, p=.00001, \eta^{2}=.47\right]$. There was no interaction $(F<1)$.

The error compensation group more often searched until finding the target than did the target-centered group $[F(1$, $\left.95)=32.88, p=.001, \eta^{2}=.25\right]$, and the distractor task decreased the proportion of completed searches $[F(1,95)=$ 76.26, $\left.p<.001, \eta^{2}=.44\right]$. We also found an interaction between group and distractor task $[F(1,95)=10.91, p=$ $.001, \eta^{2}=.1$; Fig. $\left.6 \mathrm{c}\right]$, with a larger decrease in success rate for the target-centered group after being distracted than for the error compensation group.

To estimate search efficiency, we divided the search distance by the success rate. The average distance required to succeed in finding the target was lower in the error compensation group $\left[F(1,95)=34.86, p<.0001, \eta^{2}=\right.$ .26]. The distractor task increased the total travel distance per success in both groups $\left[F(1,95)=155.98, p<.0001, \eta^{2}\right.$ $=.62]$, and there was an interaction $[F(1,95)=11.12, p=$ $.001, \eta^{2}=.1$; Fig. $\left.6 \mathrm{~d}\right]$, because the decrease with a distractor was more pronounced in the target-centered group than in the error compensation group.

Cache retrieval task

We were interested in whether the strategy in the edgefollowing task and the order of the tasks had an effect on 
Fig. 6 Comparison of error compensation and targetcentered strategies. (a) Subjects in the error compensation group more often turned toward the target than did subjects in the target-centered group. (b) Subjects in the error compensation group less often changed the direction of search and backtracked over parts of the line that they had already searched. The error compensation strategy was more efficient. (c) Subjects in the error compensation group more often succeeded in finding the target, whereas subjects in the target-centered group more often abandoned search. (d) Efficiency was measured by dividing the total distance traveled in all trials during both search and backtracking by the number of successes
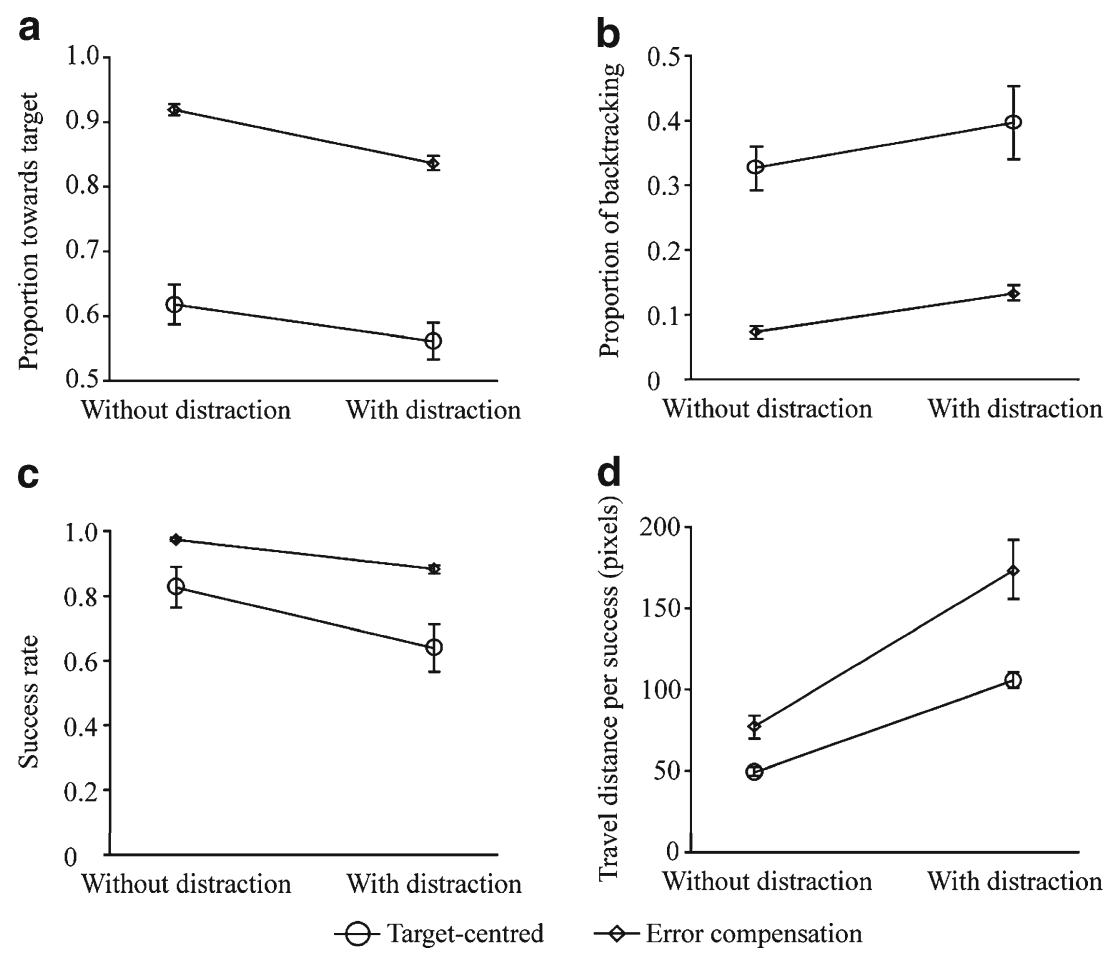

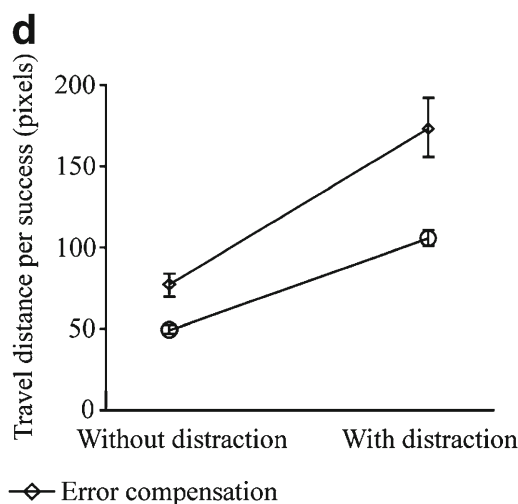

the search behavior. First, we estimated precision by how widely the first search coordinates of each subject were scattered - that is, by the standard deviations of each subject's search coordinates (Fig. 3). The precision of memory lacked any sex differences, $F<1$. We pooled the data by sex because no interaction reached significance. Subjects' precision was worse after the distractor task $[F(1,95)=129.29$, $p<.0001, \eta^{2}=.58$; Fig. 7a]. The target-centered group's search coordinates were more widely scattered than were those of the error compensation group $[F(1,95)=9.73, p=$ $\left..002, \eta^{2}=.09\right]$. Furthermore, the target-centered subjects were more affected in their precision by the distractor task than were the error compensation group [interaction of group and distraction: $\left.F(1,95)=6.71, p=.011, \eta^{2}=.07\right]$. We also found a main effect of order, with the subjects who had the edge-following task first showing less scattered search coordinates $\left[F(1,95)=5.56, p=.02, \eta^{2}=.06\right]$, but no interaction between group and order emerged $[F(1,95)=$ $\left.1.28, p=.26, \eta^{2}=.01\right]$. There was also no interaction between group, order, and distractor $[F(1,95)=2.32, p=$ $\left..14, \eta^{2}=.02\right]$. Thus, previous experience with searching on the screen made subjects more precise overall.

Is search influenced by distraction, strategy, and order? After being distracted, subjects searched longer; that is, the main effect of distraction was significant $[F(1,95)=23.55$, $\left.p<.001, \eta^{2}=.2\right]$. Error compensation subjects searched more than did the target-centered subjects $[F(1,95)=7.52$, $\left.p=.007, \eta^{2}=.07\right]$, but no interaction between distractor and group emerged $\left[F(1,95)=2.37, p=.13, \eta^{2}=.02\right]$. We also found no main effect of order $\left[F(1,95)=1.86, p=.18, \eta^{2}=\right.$
.02]. However, we found an interaction between distractor and order $\left[F(1,95)=5.87, p=.017, \eta^{2}=.06\right]$ in which those subjects who had the edge-following task first searched significantly longer after being distracted than did subjects who started with the cache retrieval task. The triple interaction was not significant, $F<1$.

\section{Discussion}

Our results show that humans can access the precision of spatial memory. This is true also for manipulating the precision internally. In the edge-following task, the 79 subjects who used the error compensation strategy provided positive evidence that they could estimate the precision of their memory by deciding how far off to the side from the remembered target position they should begin to search. In principle, the 20 who used the target-centered strategy might have demonstrated that they knew the precision of their memories by making more choices before reversing the direction of search after they had been distracted. However, too many subjects abandoned trials rather than reverse, so any such measurement would have been biased. We have no evidence whether those using the target-centered strategy assessed the precision of their memory in the edge-following task. The people who used the error compensation strategy searched more efficiently, and more often found the target.

In the cache retrieval task, 83 out of 99 searched longer after a distractor task had reduced the precision of spatial memory. Here, target-centered subjects (as identified in the 


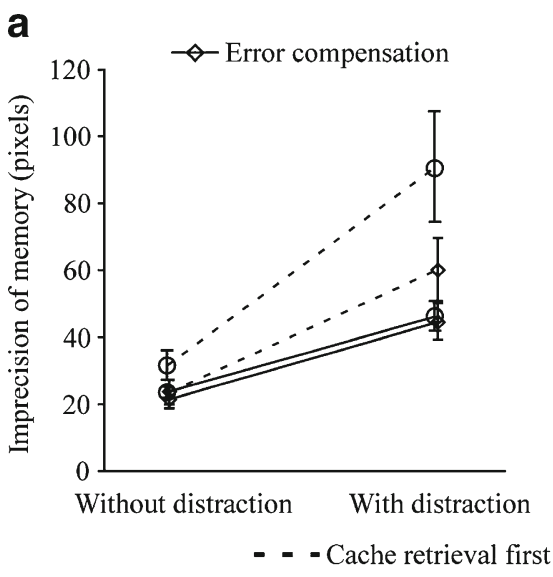

Fig. 7 Precision of memory and search effort in the cache retrieval task as a function of strategy choice in the edge-following task and task order. (a) Subjects who chose the target-centered strategy in the edgefollowing task had less precise memory in the cache retrieval task, if

edge-following task) searched less than did subjects who used the error compensation strategy in the edge-following task, and target-centered subjects also did not adjust search by their precision.

\section{Access to knowledge of the precision of spatial memory}

Information on the precision of spatial knowledge may be accessed for decision making. The decisions on where to start or when to stop searching can be modulated by previous experience. People who have their attention drawn to the importance of precision by a task in which precision seems easy to access later find it easier to access the same information in a task in which that information would otherwise make little difference. Although we used verbal reports for the initial division into groups, measures that did not rely on verbal reports discriminated well between groups and could be used for the identification of search strategies. Conceptual replications of these experimental paradigms should be well suited to comparative work, even though the details of the procedure usually would need extensive adaptation (see Chapuis \& Varlet, 1987, and Wolf \& Wehner, 2005, for examples).

The cache retrieval task is a specific example of the more general problem of how much effort to invest in an activity when feedback is delayed. A study by Falk, Lipson, and Konold (1994) suggested that some people are capable of at least some of the computations necessary for the adjustment of investment in search. Falk et al. compared humans' short- and long-term probability assessments of an object's being within a finite search space (e.g., Is a letter in the next drawer in a desk? vs. Is the letter there at all?). Out of 61 subjects, 35 correctly indicated that the chances of the letter being

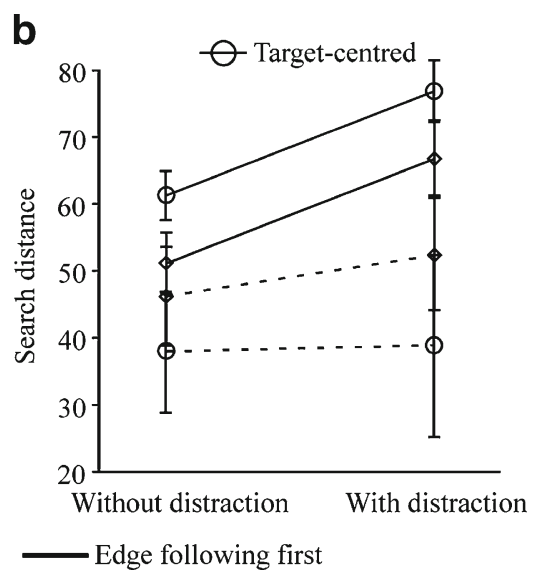

cache retrieval was their first task. Subjects in both groups remembered less precisely after being distracted. (b) Most subjects in both groups searched more after being distracted, but target-centered subjects starting with the cache retrieval task did not search longer

in the desk at all decreased with each failure to find the letter, 27 worked out that the chance of the letter being in the next drawer increased with each failure, but only 12 subjects put forward the correct combination of short- and long-term probability assessments. Falk et al. did not ask their subjects to assess the expected cost or the point at which a search should be abandoned, so we do not know how subjects might have used their probability estimates.

\section{Information flow and degree of modularity}

The experiments provided evidence both for shared, general processes and for some modularity. Comparing subjects who used different search strategies, we found that the target-centered group not only failed to use information on the precision of memory in the edgefollowing task, they also showed less precise spatial memory in the cache retrieval task. This relationship between strategy choice in one task and precision of memory in the other indicates some shared processes. For lack of other tests of spatial ability, we cannot say what the common processes may be.

However, strategy choice in the edge-following task did not predict how much the distractor task would increase search effort in the cache retrieval task. That independence of search effort in cache retrieval from strategy choices in edge following is consistent with some degree of modularity. The surprising feature of search effort was that the target-centered group, which showed no sign of accessing knowledge of precision in the edge-following task, did access that information in the cache retrieval task.

How modular is the access to knowledge of precision? For the error compensation group, task order should have an effect if access is only mildly modular. We did find the predicted dependence of search effort on task order. One 
might expect that fatigue from first completing the edgefollowing task would increase the effectiveness of the distractor task during cache retrieval, and that the resulting greater loss of precision after distraction would be more noticeable. However, our analysis showed evidence not of fatigue causing a greater loss of precision after distraction, but of practice causing a smaller loss of precision. How did that smaller loss of precision lead to a greater increase in search effort? It is plausible that exposure to the edgefollowing task triggered access to knowledge of precision and primed access to the same information in the cache retrieval task.

Performance in the two tasks was clearly not completely independent, so the system is not extremely modular. The interaction of task order with the effect of distraction was also inconsistent with a very general system. The effect of task order on search behavior best fit an intermediate level of modularity.

Part of our interest in interactions between tasks is based on the idea that one important element of cognitive sophistication, besides what computations can be carried out, is how well information processing is integrated (Rozin, 1976). We used the same stimulus presentation and manipulation of precision, then tested the use of knowledge of precision in two computationally similar tasks. Our results indicated that even in humans, probably the greatest cognitive generalists on the planet, knowledge of precision is not equally easily accessible to all processes. If Rozin is right that part of cognitive sophistication lies in greater integration of information flow and processing across domains, and if even in humans that integration is not complete, then we may expect less integration in at least some domains in many other species.

\section{Conclusion}

The three experiments showed that humans do not behave optimally in all conditions. Trommershäuser et al. (2008) reviewed differences between motor tasks and economic tasks, and suggested the performance differences might be due to the ways that statistical information is acquired. Once people have learned the relevant probability distributions, they can immediately apply this knowledge when it is relevant to a new task. Our results suggest that the type of previous experience can influence whether there is transfer. In an edge-following task, many humans will compensate for the uncertainty. However, we found that for deciding when to stop an investment, humans do not automatically use the precision of knowledge, but they can be reminded of the relevance of precision.
Author note We thank D. Derdikman, P. J. B. Girao, S. D. Healy, A. McGregor, I. Nedelcu, N. Pracic, T. Salte, K. H. Teigen, A. Chiu Werner, and $\mathrm{H}$. Wolf for comments on previous drafts or parts of the manuscript. We complied with British, German, and Norwegian regulations regarding experiments with humans.

\section{References}

Atkins, J. E., Fiser, J., \& Jacobs, R. A. (2001). Experience-dependent visual cue integration based on consistencies between visual and haptic percepts. Vision Research, 41, 449-461. doi:10.1016/ S0042-6989(00)00254-6

Biegler, R., McGregor, A., Krebs, J. R., \& Healy, S. D. (2001). A larger hippocampus is associated with longer-lasting memory. Proceedings of the National Academy of Sciences, 96, 69416944. doi:10.1073/pnas.121034798

Brown, M. F. (1992). Does a cognitive map guide choices in the radialarm maze? Journal of Experimental Psychology Animal Behavior, 18, 56-66. doi:10.1037/0097-7403.18.1.56

Chapuis, N., \& Varlet, C. (1987). Short cuts by dogs in natural surroundings. Journal of Experimental Psychology, 39B, 49-64. doi:10.1080/14640748708402251

Cheng, K., Collett, T. S., Pickhard, A., \& Wehner, R. (1987). The use of visual landmarks by honeybees: Bees weight landmarks according to their distance from the goal. Journal of Comparative Physiolology A, 161, 469-475. doi:10.1007/BF00603972

Ernst, M. O. (2007). Learning to integrate arbitrary signals from vision and touch. Journal of Vision, 7(5), 7. doi:10.1167/7.5.7

Ernst, M. O., \& Banks, M. (2002). Humans integrate visual and haptic information in a statistically optimal fashion. Nature, 415, 429433. doi: $10.1038 / 415429 a$

Falk, R., Lipson, A., \& Konold, C. (1994). The ups and downs of the hope function in a fruitless search. In G. Wright \& P. Ayton (Eds.), Subjective probability (pp. 353-377). Chichester: Wiley.

Gatty, H. (1999). Finding your way without map or compass. Mineola: Dover.

Glassman, R. B., Garvey, K. J., Elkins, K. M., Kasal, K. L., \& Couillard, N. L. (1994). Spatial working memory score of humans in a large radial maze, similar to published score of rats, implies capacity close to the magical number $7 \pm 2$. Brain Research Bulletin, 34, 151-159. doi:10.1016/0361-9230(94)90012-4

Glimcher, P. W. (2003). Decisions, uncertainty and the brain: The science of neuroeconomics. Cambridge: MIT Press.

Glimcher, P. W. (2005). Indeterminacy in brain and behavior. Annual Review of Psychology, 56, 25-56. doi:10.1146/ annurev.psych.55.090902.141429

Huttenlocher, J., Hedges, L. V., \& Duncan, S. (1991). Categories and particular: Prototype effects in estimating spatial locations. Psychology Review, 98, 352-376. doi:10.1037/0033-295X.98.3.352

Kahneman, D., Slovic, P., \& Tversky, A. (1982). Judgment under Uncertainty: Heuristics and biases. Cambridge: Cambridge University Press.

Knill, D. C., \& Pouget, A. (2004). The Bayesian brain: The role of uncertainty in neural coding and computation. Trends in Neurosciences, 27, 712-719. doi:10.1016/j.tins.2004.10.007

Körding, K. P., \& Wolpert, D. M. (2006). Bayesian decision theory in sensorimotor control. Trends in Cognitive Sciences, 10, 319-326. doi:10.1016/j.tics.2006.05.003

Macmillan, N. A., \& Creelman, C. D. (1991). Detection theory: A user's guide. Cambridge: Cambridge University Press.

Perkins, C. C., \& Weyant, R. G. (1958). The interval between training and test trials as a determiner of the slope of generalization gradients. Journal of Comparative Physiology Psychology, 51, 596-600. doi:10.1037/h0042550 
Pfuhl, G., Tjelmeland, H., \& Biegler, R. (2011). Precision and reliability in animal navigation. Bulletin of Mathematical Biology, 73, 951-977. doi:10.1007/s11538-010-9547-y

Pfuhl, G., Tjelmeland, H., Molden, S., \& Biegler, R. (2009). Optimal cache search depends on precision of spatial memory and pilfering, but what if that knowledge is not perfect? Animal Behaviour, 78, 819-828. doi:10.1016/j.anbehav.2009.06.014

Pouget, A., Dayan, P., \& Zemel, R. S. (2003). Inference and computation with population codes. Annual Review of Neuroscience, 26 , 381-410. doi:10.1146/annurev.neuro.26.041002.131112

Roach, N. W., Heron, J., \& McGraw, P. V. (2006). Resolving multisensory conflict: A strategy for balancing the costs and benefits of audio-visual integration. Proceedings of the Royal Society B, 273, 2159-2168. doi:10.1098/rspb.2006.3578

Rozin, P. (1976). The evolution of intelligence and access to the cognitive unconscious. In J. A. Sprague \& A. N. Epstein (Eds.), Progress in psychobiology and physiological psychology, vol. 6 (pp. 245-280). New York: Academic Press.

Sahani, M., \& Dayan, P. (2003). Doubly distributional population codes: Simultaneous representation of uncertainty and multiplicity. Neural Computation, 15, 2255-2279.
Thomas, D. R., \& Lopez, L. J. (1962). Effects of delayed testing on generalization slope. Journal of Comparative Physiology Psychology, 55, 541-544. doi:10.1037/ h0046103

Trommershäuser, J., Maloney, L., \& Landy, M. S. (2008). Decision making, movement planning and statistical decision theory. Trends in Cognitive Sciences, 12, 291-297. doi:10.1016/ j.tics.2008.04.010

Wehner, R., \& Srinivasan, M. V. (1981). Searching behaviour of desert ants, genus Cataglyphis (Formicidae, Hymenoptera). Journal of Comparative Physiology. A, 142, 315-338.

White, K. G., \& Wixted, J. T. (1999). Psychophysics of remembering. Journal of the Experimental Analysis of Behavior, 71, 91-113. doi:10.1901/jeab.1999.71-91

Wolf, H., \& Wehner, R. (2005). Desert ants compensate for navigation uncertainty. Journal of Experimental Biology, 208, 4223-4230. doi:10.1242/jeb.01905

Zar, J. H. (1999). Biostatistical analysis. Upper Saddle River: Prentice-Hall.

Zhang, W., \& Luck, S. J. (2008). Discrete fixed-resolution representation in visual working memory. Nature, 453, 233-236. doi:10.1038/nature06860 